\title{
TU/e EN⿴HONE

\section{Model-based predictive control scheme for cost optimization and balancing services for supermarket refrigeration Systems}

\section{Citation for published version (APA):}

Weerts, H. H. M., Shafiei, S. E., Stoustrup, J., \& Izadi-Zamanabadi, R. (2014). Model-based predictive control scheme for cost optimization and balancing services for supermarket refrigeration Systems. In E. Boje, \& X. Xia (Eds.), Proceedings of the 19th IFAC World Congress of the International Federation of Automatic Control, (IFAC'14), 24-29 August 2014, Cape Town, South Africa, part 1 (pp. 975-980). Pergamon. https://doi.org/10.3182/20140824-6-ZA-1003.01056

DOI:

10.3182/20140824-6-ZA-1003.01056

Document status and date:

Published: 01/01/2014

\section{Document Version:}

Publisher's PDF, also known as Version of Record (includes final page, issue and volume numbers)

\section{Please check the document version of this publication:}

- A submitted manuscript is the version of the article upon submission and before peer-review. There can be important differences between the submitted version and the official published version of record. People interested in the research are advised to contact the author for the final version of the publication, or visit the $\mathrm{DOI}$ to the publisher's website.

- The final author version and the galley proof are versions of the publication after peer review.

- The final published version features the final layout of the paper including the volume, issue and page numbers.

Link to publication

\section{General rights}

Copyright and moral rights for the publications made accessible in the public portal are retained by the authors and/or other copyright owners and it is a condition of accessing publications that users recognise and abide by the legal requirements associated with these rights.

- Users may download and print one copy of any publication from the public portal for the purpose of private study or research.

- You may not further distribute the material or use it for any profit-making activity or commercial gain

- You may freely distribute the URL identifying the publication in the public portal.

If the publication is distributed under the terms of Article 25fa of the Dutch Copyright Act, indicated by the "Taverne" license above, please follow below link for the End User Agreement:

www.tue.nl/taverne

Take down policy

If you believe that this document breaches copyright please contact us at:

openaccess@tue.nl

providing details and we will investigate your claim. 


\title{
Model-Based Predictive Control Scheme for Cost Optimization and Balancing Services for Supermarket Refrigeration Systems
}

\author{
H.H.M. Weerts * S. E. Shafiei ${ }^{* *}$ J. Stoustrup ${ }^{* *}$ \\ R. Izadi-Zamanabadi ${ }^{* * *}$ \\ * Department of Electrical Engineering, Eindhoven University of \\ Technology, Eindhoven, Netherlands, (e-mail: \\ h.h.m.weerts@student.tue.nl). \\ ** Automation and Control, Department of Electronic Systems, Aalborg \\ University, Aalborg, Denmark, (e-mail: \{ses, jakob\}@es.aau.dk) \\ *** Danfoss A/S, 6430 Nordborg, Denmark, (e-mail: \\ roozbeh@danfoss.com)
}

\begin{abstract}
A formulation of model predictive control for supermarket refrigeration systems is proposed to facilitate the regulatory power services as well as energy cost optimization of such systems in the smart grid. Nonlinear dynamics existing in large-scale refrigeration plants challenges the predictive control design. It is however shown that taking into account the knowledge of different time scales in the dynamical subsystems makes possible a linear formulation of a centralized predictive controller. A realistic scenario of regulatory power services in the smart grid is considered and formulated in the same objective as of a cost optimization one. A simulation benchmark validated against real data and including significant dynamics of the system is employed to show the effectiveness of the proposed control scheme.
\end{abstract}

\section{INTRODUCTION}

Stability of the electricity grid is threatened by the increasing amount of intermittent energy sources such as wind power as stated by Milligan and Kirby [2010]. The transmission system operator (TSO) will have to utilize more and more ancillary services such as spinning reserves to keep the grid balanced. A smart grid, where both production and consumption will be efficiently managed, would greatly facilitate the grid stability reserve by incorporating the demand side management services. Equipping energy consuming applications with smart control algorithms can modify electricity consumption profiles to meet grid requirements.

According to Heffner et al. [2007] ancillary services can be divided into 3 main types: normal services, contingency services and other services. Normal services are mainly used to compensate minute to minute fluctuations. Contingency services are used to compensate for unpredicted drops or rises in power consumption or production. The latter service is addressed in the present paper.

Dynamic Demand Response (DDR) is a control strategy used to manage the electricity consumption profile. The first way to implement DDR is to optimize consumption based on real-time electricity prices. The second way is to directly manage the consumption profile. Both strategies consist of 2 levels of design: a high level to pass grid requirements on to the application, and a low level to locally provide the requested balancing services. This paper focuses on the low level for both real-time pricing and direct management.
A good candidate for DDR implementation needs to have the ability to store large amounts of energy to shift electricity consumption in time. Heat capacity is a natural energy storage available almost anywhere, but rarely exploited as such. The potential of several types of thermal storage possibilities (Heat storage, ventilation, refrigeration) is shown in Stadler [2008]. Refrigeration systems have a large potential for storing energy and shifting loads as shown in Goli et al. [2011].

An excellent choice for predicting future plant behavior and handling input and state constraints is Model Predictive Control (MPC), Maciejowski [2002]. Optimal power consumption or storage of energy is achieved by minimization of a cost function over a prediction horizon, Rawlings and Amrit [2009]. A strategy where an MPC is used as a supervisory controller to generate references for local controllers is shown by Shafiei et al. [2013b] for operating cost minimization in refrigeration systems. Hovgaard et al. [2012] presents an MPC algorithm to optimize cost of operation as well as providing normal ancillary services, where a non-convex optimization problem is put forward. Direct control implementation of single vapor compression cycle (VCC) is presented in Pedersen et al. [2013] where a simple bucket model is introduced for energy modeling of the system. It is shown that how the flexible power consumption of refrigeration systems can be employed for minimization of the fossil fuel production sources in a small grid.

The problem of operating cost minimization and grid balancing services for refrigeration systems are mainly investigated separately in different researches. In this paper a formulation of MPC is proposed to meet both of the 
above objectives simultaneously in a realistic scenario. Balancing services are provided in the framework of $i n$ stantaneous contingency reserves, Heffner et al. [2007], by reducing power consumption during contingency events after optimally pre-cooling the cold reservoirs. The cost of operation is reduced by taking a price signal into account in the cost optimization function. It is shown that the power consumption of the SRS can be highly reduced (even close to zero consumption) for an hour without violating food temperature constraints. At the same time, cost of operation is reduced by $6.3 \%$ for a day of operation.

\section{SUPERMARKET REFRIGERATION SYSTEMS}

\subsection{Booster configuration}

The fridge and freezer display cases are cooled by evaporators where refrigerant ( $\mathrm{CO}_{2}$ in this particular system) evaporates and absorbs thermal energy. A schematic view of an SRS with booster configuration is shown in Figure 1. The configuration includes medium and low temperature (MT and LT) cooling sections each contains several display cases and a rack of compressors. Starting at the receiver (REC), both liquid and vapor forms of the refrigerant flow into the system. Vapor flows through the bypass valve (BPV) directly to the compressor rack. Liquid flows to the expansion valves EV_MT and EV_LT, where local controllers control the flow to regulate the temperature in low and medium temperature display cases. Refrigerant absorbs heat from the cold room when evaporating (EVAP_MT, EVAP_LT). A compressor rack (COMP_LO) increases the pressure of the LT units. All refrigerant (EVAP_MT, BPV, COMP_LO) is sucked into the suction manifold at point 5.Pressure is increased by the high stage compressor rack (COMP_HI). The refrigerant condenses in the condenser losing the absorbed heat to the surroundings. Liquid refrigerant is collected by the receiver, closing the circle.

\subsection{System dynamics}

Inside the display cases heat is transferred from the food to cooled air $\left(\dot{Q}_{\text {food }}\right)$, and from the cooled air to the evaporator $\left(\dot{Q}_{e}\right)$, where the latter is also known as the cooling capacity. A heat load caused by the supermarket indoor temperature is formulated as a disturbance $\left(\dot{Q}_{l o a d}\right)$. We assume that air and food temperatures are homogenous and we use energy balances to derive the dynamical equations.

$$
\begin{aligned}
M C p_{\text {food }} \frac{d T_{\text {food }}}{d t} & =-\dot{Q}_{\text {food }} \\
M C p_{\text {air }} \frac{d T_{\text {air }}}{d t} & =\dot{Q}_{\text {load }}+\dot{Q}_{\text {food }}-\dot{Q}_{e} \\
\dot{Q}_{\text {food }} & =U A_{\text {food }}\left(T_{\text {food }}-T_{\text {air }}\right) \\
\dot{Q}_{\text {load }} & =U A_{\text {load }}\left(T_{\text {indoor }}-T_{\text {air }}\right) \\
\dot{Q}_{e} & =U A_{e}\left(T_{\text {air }}-T_{e}\right)
\end{aligned}
$$

where $M C p$ denotes mass multiplied with heat capacity, $\mathrm{UA}$ is the overall heat transfer coefficient, and $T_{e}$ is the evaporation temperature which is a function of the suction pressure. The heat transfer coefficient of the evaporator

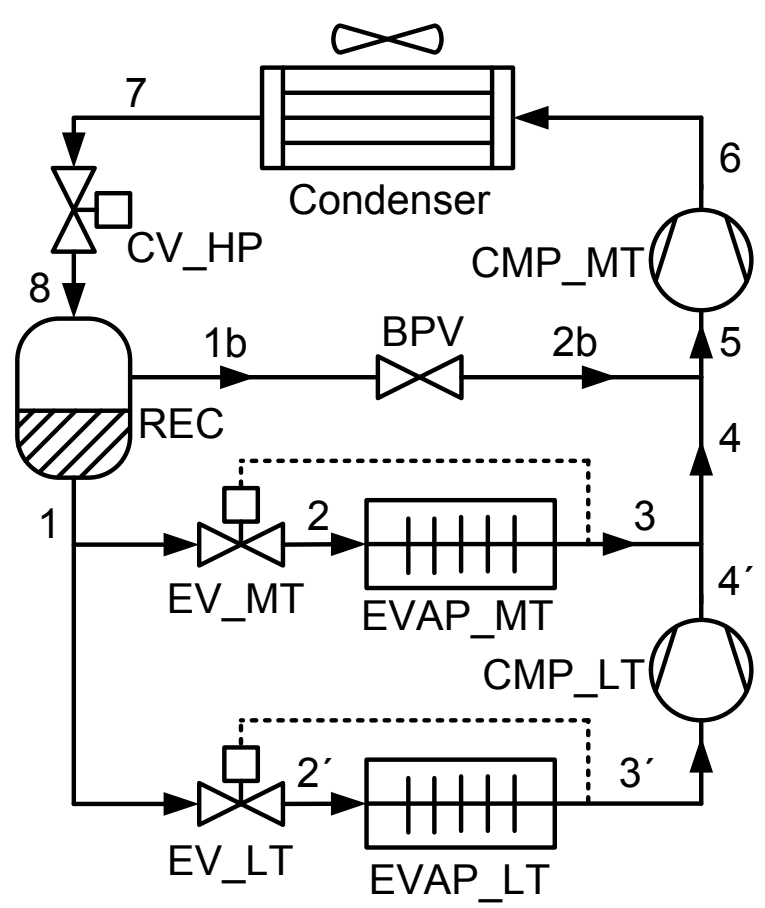

Fig. 1. schematic view of the SRS system

depends on the refrigerant mass in the evaporator is described as a linear function of the latent mass as

$$
\begin{aligned}
U A_{e} & =k_{m} M_{r} \\
\frac{d M_{r}}{d t} & =\dot{m}_{r, \text { in }}-\dot{m}_{r, \text { out }}
\end{aligned}
$$

where $k_{m}$ is a constant, and the latent mass in the evaporator is restricted to $0 \leq M_{r} \leq M_{r, \max }$.

$$
\dot{m}_{r, i n}=O D \cdot K v A \sqrt{\rho_{\text {suc }}\left(P_{\text {rec }}-P_{\text {suc }}\right)}
$$

Inflow of mass is determined by opening degree of the expansion valve (OD) and suction pressure. Outflow depends on cooling capacity and suction pressure.

$$
\dot{m}_{r, \text { out }}=\frac{\dot{Q}_{e}}{\Delta h_{l g}}
$$

where $K v A$ is the valve constant, $P_{r e c}$ is the receiver pressure assumed constant here and $\rho_{s u c}$ and $\Delta h_{l g}$ nonlinear functions of the suction pressure (or equivalently the evaporation temperature). The suction pressure is regulated by a local controller, and we assign a fixed set-point to it in this work.

Electrical power consumption from the compressor racks depends on the enthalpy difference at the inlet and outlet.

$$
\dot{W}_{c}=\frac{1}{\eta_{m e}} \dot{m}\left(h_{o, c}-h_{i, c}\right)
$$

where $\eta_{m e}$ is the efficiency of mechanical/electrical conversion, $\dot{m}$ the massflow through the compressor. , and $h_{i, c}$ and $h_{o, c}$ are the inlet and outlet enthalpies, respectively. The nonlinear thermophysical properties of the refrigerant (e.g. enthalpies) are calculated by the software package "RefEqns" Skovrup [2000]. The coefficient of performance $(\mathrm{COP})$ is a measure of efficiency of the cooling system, where a higher COP means less electricity is required to provide the same cooling capacity.

$$
C O P=\frac{\dot{Q}_{e, t o t}}{\dot{W}_{t o t}}
$$


The system is obviously non-linear and later on we will show how we can formulate a linear MPC from these dynamics.

\section{PROBLEM STATEMENT}

Stability of wind powered electricity grids is threatened by either sudden increased loads or drops in power generation according to Milligan and Kirby [2010]. Increases in generation or drops in load are less of a stability threat, but they are an economically unattractive situation. Since the former is more of a concern and a more challenging problem, we will focus on that situation in this paper. Immediate reaction to these contingency events without prior knowledge of these happening can be achieved when constantly pre-cooling the SRS. Continuous lower temperatures will result in more losses and therefore a higher cost. Pre-cooling right before the event happens would be cheaper, but predicting the exact time of the event is difficult and not the goal of this research. We therefore assume that either a TSO or aggregator can predict a time interval with a high probability of a contingency event happening, based on weather models and load history data. The SRS will only pre-cool before these intervals and therefore waste less energy. The drawback is that an event might happen and not be predicted beforehand, the SRS will then not be able to provide service.

The most important characteristics of load response are response speed, duration and cost. Since we are aiming for the system to be used for instantaneous contingency reserves, we need a fast response speed. The duration of the load response should be long to give more support to the grid. Optimizing the cost of pre-cooling and standby time is always important and could go hand in hand with cost optimization of the whole system. It could be called optimal if not more energy is stored than needed, seen by the temperatures to reach normal levels when the event ends.

An example of the behavior we want to see is shown in Figure 2. The upper plot shows the air and food temperatures, and the lower plot shows the power consumption. In this example there is a 4 hours notice before the high likelihood interval where the food is cooled in an optimal way. The food temperature remains almost unchanged during the standby interval. During the standby, the air temperature is controlled in order to maintain the food temperature at a predefined constant level. The power consumption drops to a low level when the contingency event actually happens (unpredicted) and the temperatures rise subsequently. The constraints imposed on the food temperature are fully respected.

\section{COST MINIMIZATION}

In order to minimize the cost of operation we have to multiply the electricity price with the power consumption and find the minimum of an objective function over the horizon $N$. The objective function is subject to the plant dynamics and constraints which will be elaborated in the sequel.

$$
J=\sum_{i=1}^{N} e_{p}(i) \dot{W}_{t o t}(i)
$$

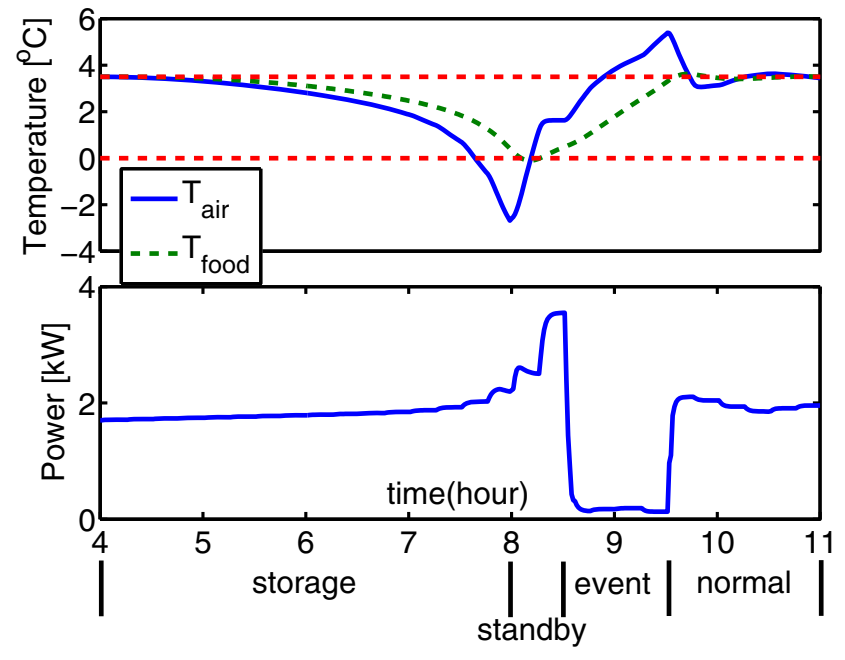

Fig. 2. Example of an favorable SRS behavior during the imbalance management.

where $e_{p}(i)$ is the electricity price and $\dot{W}_{t o t}(i)$ is the total power consumption of the compressor racks. We can model the power consumption by using (11) to replace power consumption by cooling capacity, however it is divided by the COP. In Shafiei et al. [2013b], it is explained that for the case where the condenser fan is fixed in a speed (usually close to maximum), the average value of the COP is highly correlated with the value of the outdoor temperature. Outdoor temperature predictions are fairly accurate and can be used to predict the COP in (13).

Other influences on the COP are evaporation temperature and mass flow out of the evaporators as stated by Shafiei et al. [2014]. The evaporation temperature is constant in this work due to the constant suction pressure. The COP changes caused by the mass flow are mainly higher frequencies than the MPC sample frequency, and the amplitude is relatively small compared to the outdoor temperature dynamics. Making the COP dependant on the mass flow would result in a non-convex problem and is not done in this work.

$$
J=\sum_{i=1}^{N} e_{p}(i) \frac{\dot{Q}_{e, t o t}(i)}{C O P(i)}
$$

Calculating the cooling capacity using (5) and (6) is both non-linear and requires the state $M_{r}$ to be known, which is however not measurable. The latent mass dynamics described by (7), (8) and (9) reach steady state after around 4 minutes. We can fairly assume the latent mass in steady-state when the sampling time is chosen longer than 4 minutes. Equation (16) shows the linear relation between the valve opening degree and the cooling capacity.

$$
\begin{aligned}
\dot{m}_{r, \text { out }} & =\dot{m}_{r, \text { in }} \\
\frac{\dot{Q}_{e}}{\Delta h_{l g}} & =O D \cdot K v A \sqrt{\rho_{s u c}\left(P_{r e c}-P_{s u c}\right)} \\
\dot{Q}_{e} & =O D \cdot K v A \cdot c_{1}
\end{aligned}
$$

where $c_{1}=\sqrt{\rho_{s u c}\left(P_{r e c}-P_{s u c}\right)} \Delta h_{l g}$ represents all the terms we assumed constant. The steady-state model results in a linear relation between cooling capacity and opening degree which we use in the objective function. 


$$
J=\sum_{i=1}^{N} e_{p}(i) \frac{\sum_{j=1}^{n_{c}} O D_{j}(i) \cdot K v A_{j}}{C O P(i)} c_{1}
$$

where $n_{c}$ is the number of cooling units.

\subsection{System dynamics}

The plant dynamics are the temperatures of food and air. The continuous state-space representation of the dynamics of the $j$ th display case is shown below.

$$
\left[\begin{array}{c}
\dot{T}_{\text {food }, j} \\
\dot{T}_{\text {air }, j}
\end{array}\right]=A_{c}\left[\begin{array}{c}
T_{\text {food }, j} \\
T_{\text {air }, j}
\end{array}\right]+B_{c}\left[\begin{array}{c}
T_{\text {indoor }} \\
O D_{j}
\end{array}\right]
$$

The disturbance $T_{\text {indoor }}$ is formulated as an input for simplicity, and the matrices $A_{c}$ and $B_{c}$ are calculated using $(1),(2),(3),(4)$ and (16). The objective function is formulated in discrete time and needs discretized dynamics. The matrices $A_{c}$ and $B_{c}$ are discretized by a zero order hold method, giving (19).

$$
\left[\begin{array}{c}
T_{\text {food }, j}(i+1) \\
T_{\text {air }, j}(i+1)
\end{array}\right]=A_{d}\left[\begin{array}{c}
T_{\text {food }, j}(i) \\
T_{\text {air }, j}(i)
\end{array}\right]+B_{d}\left[\begin{array}{c}
T_{\text {indoor }}(i) \\
O D_{j}(i)
\end{array}\right]
$$

\subsection{Constraints}

For the sake of food safety, the following constraints are imposed on the food temperatures.

$$
\begin{array}{r}
0<T_{\text {food }, M T}(i)<3.5 \\
-26<T_{\text {food }, L T}(i)<-18
\end{array}
$$

These constraints have to be satisfied in the prediction of the MPC even during an event where no cooling is applied. This forces the MPC to start pre-cooling before an event which is an optimal strategy due to cost optimization.

The system has physical limitations in the opening degree of the valves and the amount of mass that can be stored in the evaporators. The mass in the evaporators is not a state in the system so we found a way to restrict the input such that the evaporators do not overflow. Using (5), (6) and (16) the input constraint is given by

$$
0<O D<\min \left(1, \frac{k_{m} M_{r, \max }\left(T_{a i r}-T_{e}\right)}{K v A c_{1}}\right)
$$

\section{REGULATING POWER SERVICES}

Using the SRS to counteract supply and demand imbalances can be done by manipulating the power consumption of the compressor racks. Surplus electricity can be counteracted by increasing the consumption without a beforehand preparation like precooling. Electricity deficit requires the compressor racks to reduce the power consumption at an unknown moment. Note that, only the interval where the contingency event might happen is assumed known. Precooling the food is needed to not violate the temperature constraints during the event.

We want to reduce the power consumption of the system during a contingency event. Introducing a constraint on the power consumption during this event would be a simple solution. It means that the system is able to deliver at least some agreed $\mathrm{kWh}$ of energy during the event. This constraint is set in the control horizon so the system can precool the display cases. As time passes this constraint will shift through the horizon to the first point and become smaller while the event is taking place.

$$
\begin{aligned}
& \dot{W}_{\text {tot }}\left(i_{\text {event }}\right)<\dot{W}_{\text {max }} \\
& i_{\text {event }}=\left[\frac{t_{\text {start }}-t}{T_{s}}, \frac{t_{\text {start }}-t}{T_{s}}+1, \cdots, \frac{t_{\text {end }}-t}{T_{s}}\right]
\end{aligned}
$$

where $t_{\text {start }}$ and $t_{\text {end }}$ are the start and end of the contingency event. $T_{s}$ is the sampletime of the controller and $t$ is the current time. The result is that $i_{\text {event }}$ is a sequence of integers which is updated at every time step for the receding horizon implementation. The MPC will automatically store energy before the event happens in an optimal way.

In order to manage the electrical power consumption by controlling the thermal dynamics of the system, we can rewrite (23) in terms of the opening degrees of the expansion valves as:

$$
\frac{\sum_{j=1}^{n_{c}} O D_{j}\left(i_{\text {event }}\right) \cdot K v A_{j}}{C O P\left(i_{\text {event }}\right)} c_{1}<\dot{W}_{\max }
$$

Not knowing when exactly the event will happen changes things to some extent. The energy has to be stored before the start of the interval to be ready all the time, a different $i_{\text {event }}$ is used for this in (26). During the interval before the event has happened, the system will be in standby and hold the achieved temperatures. When the event actually happens, energy will be released like before.

$$
i_{\text {event }}=\left[\frac{t_{\text {int }}-t}{T_{s}}, \frac{t_{\text {int }}-t}{T_{s}}+1 \cdots \frac{t_{\text {int }}+t_{\text {length }}-t}{T_{s}}\right]
$$

where $t_{\text {int }}$ is the starting time of the interval and $t_{\text {length }}$ is the duration of the event.

\section{SIMULATION RESULTS}

Simulations are performed on a benchmark system developed in MATLAB using a validated model described in Shafiei et al. [2013a]. The benchmark features 7 medium temperature display cases and 4 low temperature display cases. Both food and air temperatures are measured in each display case, and the input we control is the opening degrees of the expansion valves. The compressor racks are modeled as a big virtual variable speed compressor to simplify the setup.

The benchmark simulates 24 hours of operation with 1 event, where the interval starts at 10:00 and the duration of the event is 1 hour. The MPC uses a 24 hour horizon and 15 minute sampling time. Outdoor temperature is a sinusoid that varies between 6 and 16 degrees celcius.

The optimization problem that should be solved at each time step for the MPC implementation includes the objective function (17) together with:

- 22 state variables representing the air and food temperatures, (19),

- 11 input variables representing opening degrees of the expansion valves,

- 11 state constraints on the food temperatures, (20) and (21),

- 11 input constraints, (22),

- 11 output constraints that are only activated during the regulating power service, (25), 
- predicted signal of the outdoor temperature,

- predicted signal of the electricity price, and

- estimated signal of the system COP.

Moreover, in order maintain the above constrained optimization problem feasible, two sets of slack variables are introduced to soften the state output constraints. Note however that the problem dimension is scaled by the value of the prediction horizon.

The first simulation uses no COP or price optimization. Temperatures of medium and low temperature units are shown in Figures 3 and 4 . The system has the interval in the prediction horizon and starts storing thermal energy slowly. Thermal storage is finished at the start of the interval at 10:00. The contingency event happens at 10:45 and lasts until 11:45. Figure 5 shows the total power consumption of the system, the power is close to 0 during the event. The temperatures respond by rising and are close to the permitted value at the end of the event which shows the optimal amount of the thermal energy storage.

One could ask what the price of this event was, we will use the price signal in Figure 6 for analysis. The baseline operational cost for 1 day is $185.04 \mathrm{DKK}$, the operational cost with an event is $183.87 \mathrm{DKK}$. The event actually reduced the cost compared to the baseline. The electricity price while storing energy was relatively low, and the price while releasing was relatively high which caused this drop in operational cost compared to the baseline. It is also possible that there is a price peak while storing energy which would cause a high cost of an event. The conclusion is that there is no 'fixed price' of an event since it is related to the electricity prices during storage and release.
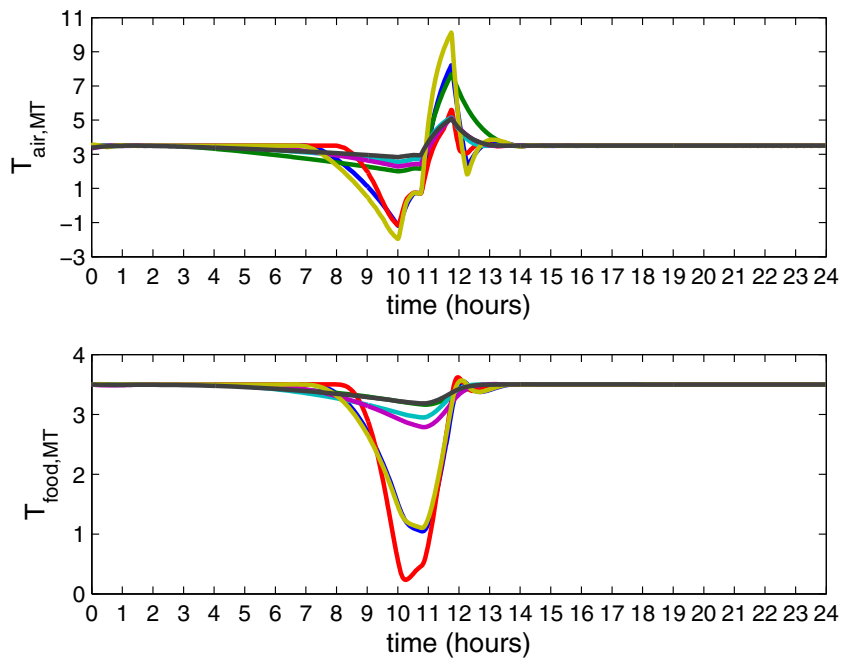

Fig. 3. Temperature response of medium temperature units

The second simulation uses COP and price optimalization, where the price signal used is the same as before. Everything else is the same as in the first simulation. Figure 7 shows the power consumption of the second simulation in red compared to the first simulation in green. The second simulation stored energy during the first 8 hours because the electricity price is low. The price is higher during the day which is when the energy is released. The total cost of the energy for this day is $172.22 \mathrm{DKK}$ which is a reduction of $11.65 \mathrm{DKK}$ or $6.3 \%$.
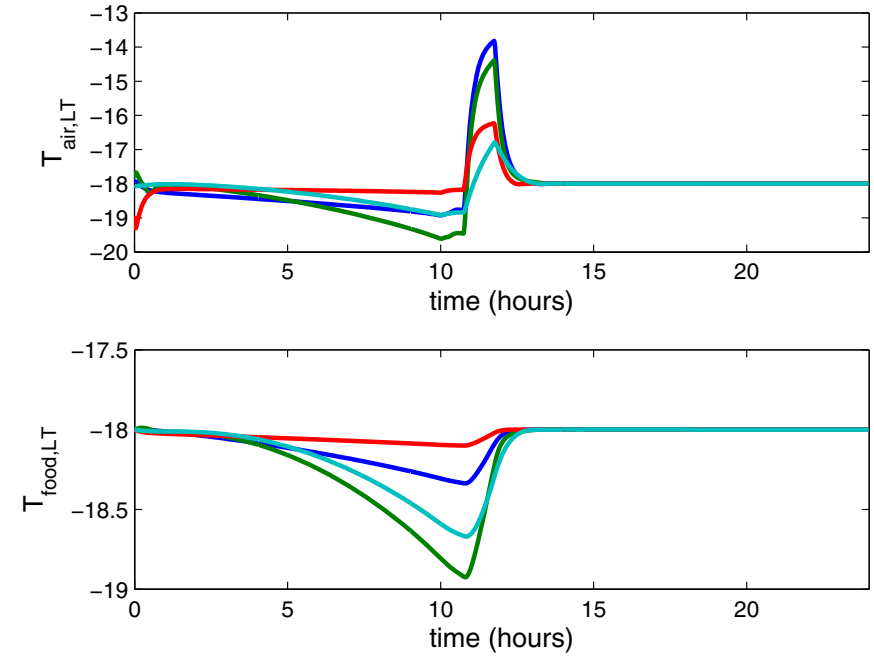

Fig. 4. Temperature response of low temperature units

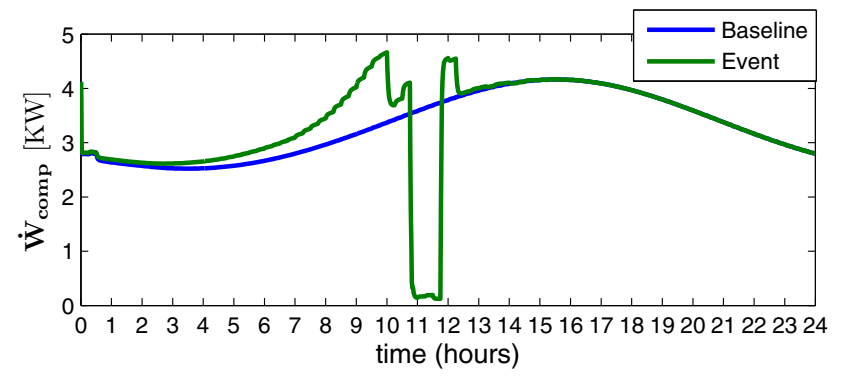

Fig. 5. Total power consumption of the SRS as baseline (blue) and during the event (green)

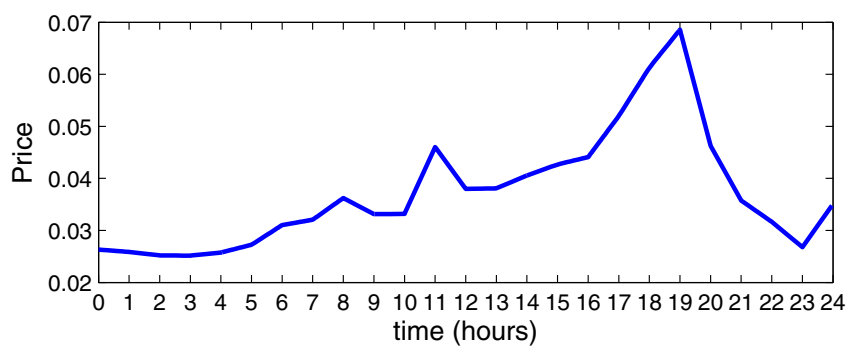

Fig. 6. Price signal used for simulation

\section{DISCUSSION}

The simulations show that it is possible to store and recover energy in the products in the SRS. In reality the thermal masses and heat transfer coefficients will not be constant, because products are sold or refilled during the day. Selling the products does not change the temperatures in the display cases, but the products might increase in temperature more rapidly due to the lower thermal mass. The sold products could be pre-cooled and this energy then can't be recovered anymore which results in additional losses. Only a small part of the products will be sold during the interval and event so these issues are likely to be small. On the other hand refilling display cases with products could change the temperatures in the display case. But products will increase slower in temperature due to the extra thermal mass, making this issue likely small. Making realistic selling and refilling scenarios, and making 


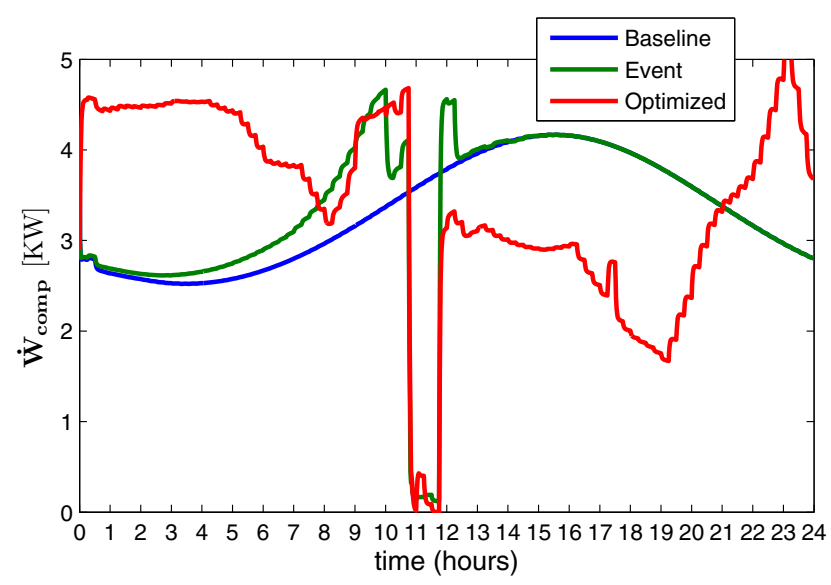

Fig. 7. Total power consumption with price and COP optimalization shown in red, without optimalization is shown in green and the baseline in blue

a suitable robust controller is needed, but outside of the scope of the project and is therefore left to future work.

\section{CONCLUSIONS}

Utilization of supermarket refrigeration systems for grid imbalance management under contingency reserve service was presented. Since the service is limited to the specific hours during a day, there is still more rooms for energy cost optimization for the off-service periods. A new MPC scheme was proposed to deal with both the balancing services and cost optimization as a central controller at the local plant level. A realistic scenario is simulated using a large-scale refrigeration benchmark. The proposed control method successfully provided the required services as well as reducing the operating cost by $6.3 \%$.

\section{ACKNOWLEDGEMENTS}

The authors wish to thank Danfoss Air-Conditioning \& Refrigeration for supporting this work.

\section{REFERENCES}

S. Goli, A. McKane, and D. Olsen. Demand response opportunities in industrial refrigerated warehouses in california. In 2011 ACEEE Summer Study on Energy Efficiency in Industry, Niagara Falls, NY, USA, July 2011.

G. Heffner, C. Goldman, B. Kirby, and M. Kintner-Meyer. Loads providing ancillary services: Review of international experience. Technical report, U.S. Department of Energy, Ernesto Orlando Lawrence Berkeley National Laboratory, May 2007.

T.G. Hovgaard, L.F.S. Larsen, K. Edlund, and J.B. Jørgensen. Model predictive control technologies for efficient and flexible power consumption in refrigeration systems. Energy, 44:105-116, 2012.

J.M. Maciejowski. Predictive Control with Constraints. Pearson Education. Prentice Hall, 2002. ISBN 9780201398236 .

Michael Milligan and Brendan Kirby. Utilizing load response for wind and solar integration and power system reliability. In WindPower, Dallas, Texas, May 2010 .
R. Pedersen, J. Schwensen, S. Sivabalan, C. Corazzol, S. E. Shafiei, K. Vinther, and J. Stoustrup. Direct control implementation of a refrigeration system in smart grid. In Proceedings of the American Control Conference, Washington DC, USA, June 2013.

J.B. Rawlings and R. Amrit. Optimizing Process Economic Performance using Model Predictive Control, pages 119138. Nonlinear Model Predictive Control: Towards New Challenging Applications, ISBN: 978-1-4614-18320. Springer, 2009.

S. E. Shafiei, H. Rasmussen, and J. Stoustrup. Modeling supermarket refrigeration systems for demand-side management. Energies, 6(2):900-920, 2013a.

S. E. Shafiei, J. Stoustrup, and H. Rasmussen. A supervisory control approach in economic mpc design for refrigeration systems. In Proceedings of the European Control Conference, Zürich, Switzerland, July 2013b.

S. E. Shafiei, J. Stoustrup, and H. Rasmussen. Model predictive control for flexible power consumption of large-scale refrigeration systems. In Proceedings of the American Control Conference, Portland, OR, USA, June 2014.

M.J Skovrup. Thermodynamic and Thermophysical Properties of Refrigerants, Version 3.00. Technical University of Denmark: Copenhagen, Denmark, 2000.

I. Stadler. Power grid balancing of energy systems with high renewable energy penetration by demand response. Utilities Policy, 16:90-98, 2008. 